\title{
AO error breakdown: anisoplanatism and bandwidth error correlation with ROKET
}

\author{
F. Ferreira ${ }^{\mathrm{a}}$, E. Gendron ${ }^{\mathrm{a}}$, G. Rousset ${ }^{\mathrm{a}}$, and D. Gratadour ${ }^{\mathrm{a}}$ \\ ${ }^{a}$ LESIA, Observatoire de Paris, PSL Research University, CNRS, Sorbonne Universités, \\ UPMC Univ. Paris 06, Univ. Paris Diderot, Sorbonne Paris Cité, 5 place Jules Janssen, 92195 \\ Meudon, France
}

\begin{abstract}
Future Extremely Large Telescope (ELT) adaptive optics (AO) systems will aim at wide field correction and high sky coverage. However, AO correction is only partial and images obtained with such systems can be improved by using post processing techniques for image inversion relying on a field-dependent point spread function (PSF). This requires an accurate knowledge of the PSF and its variations over the science field. The PSF estimation involves identifying the various sources of wave-front error from the AO system telemetry. Estimating and disentangling these error contributors is an issue due to the propagation and filtering process in the AO loop, and numerical simulations are a good way to address it. Moreover, assumptions such as statistical independence are usually made on the error contributors to perform PSF reconstruction. A detailed estimation tool is a good way to study the behaviour of AO error breakdown contributors. However, each simulation step at the ELT scale is very demanding in terms of computing power and data flow and the simulation of a long exposure PSF requires several thousands of instantaneous images. Moreover, the error breakdown estimation usually requires to perform several simulations to identify the different contributors. To lead this study, we used COMPASS (COMputing Platform for Adaptive opticS Systems), an end-to-end simulation tool especially developed to reach acceptable simulation time for ELT scales using GPU accelerators. We have developed in COMPASS an estimation tool called ROKET (erROr breaKdown Estimation Tool) that provides a comprehensive error breakdown as the output of a single simulation run. This is done by using known internal quantities computed by the simulation to estimate on the fly the impact of those errors on the AO performance without interrupting the main AO loop. This error budget returns, for example, the contribution of tomography (reconstruction) error, aliasing, fitting, wavefront sensing noise, or loop delay temporal error. As the outputs of ROKET are the temporal buffers of each error contributor, we are able to study their behaviours and to take into account the possible correlation between them. We have, as well, implemented a GPU accelerated algorithm to reconstruct ELT scale PSF efficiently, so that the impact of the error contributors and their correlation could be seen on the PSF. After a description of the error breakdown to be computed, we outline the estimation methods and its implementation in COMPASS. Then, we will use this estimation tool on ELT cases to study possible correlation between some contributors, especially correlation between anisoplanatism and temporal errors.
\end{abstract}

Keywords: Adaptive optics, Numerical simulation, Error budget

\section{INTRODUCTION}

The resolution of the images obtained with ground based telescopes are limited by the effects of atmospheric turbulence. It generates optical phase aberrations, that need to be corrected in order to retrieve the diffraction limit of the telescope. Without any compensation, image resolution in the visible is similar to the one obtained with a diffraction-limited telescope of a diameter not greater than a few tens of centimetres. To handle that, Adaptive Optics (AO) systems use wavefront sensors (WFS) to measure the wavefront deformation and control a deformable mirror (DM) to flatten the wavefront in real-time for the scientific camera.

Unfortunately, AO systems do not achieve a perfect compensation. Some residual wavefront deformations still degrade the image quality to a level that depends on the observing and atmospheric conditions (source brightness,

Further author information: (Send correspondence to F. Ferreira)

F. Ferreira: E-mail: florian.ferreira at obspm.fr, Telephone: +33 (0)1 45077178 
seeing, etc.). The contrast of the acquired image can be further improved using post-processing techniques such Point Spread Function (PSF) deconvolution algorithms. In order to use efficiently this kind of techniques, an estimation of the corrected PSF is required. In the most general case the corrected PSF has to be estimated from the real-time data of the AO system, due to the lack of any bright point-like object within the field. This estimation involves a characterization of the error contributors of the AO system, especially the covariance matrix of those errors. ${ }^{1-3}$ To be able to compute this matrix from loop data, error contributors are often supposed to be statistically independent.

Estimating each error contributor independently from the other is a tricky process because of the AO loop filtering process, but numerical simulations provide a solution to address it. However, an end-to-end simulation of an AO system at the Extremely Large Telescope (ELT) scale is very demanding in terms of computing power and data flow. To estimate accurately the error budget of the AO system, several thousands of iterations of the AO loop are required to provide a proper statistical convergence. Moreover, classical methods to obtain the full error breakdown usually require several of those simulations. It leads to a very time-consuming process.

In this paper, we use a new estimation tool, called ROKET (erROr breaKdown Estimation Tool), that provides a comprehensive error breakdown as an output of a single run of an AO system simulation. ${ }^{4}$ It is developed as a module of the end-to-end Graphics Processing Units (GPU) based simulation tool COMPASS (COMputing Platform for Adaptive opticS Systems). ${ }^{5}$ After a brief description of ROKET, we will use it to study potential error correlation at an ELT scale system. Then, we will focus on the correlation between anisoplanatism and bandwidth errors by proposing a way to model it in a pseudo-analytical way. We will finally show the results obtained with this model at the scale of the E-ELT.

\section{ROKET: ERROR BREAKDOWN ESTIMATION TOOL}

ROKET is the name given to a specific feature that we have developed within the end-to-end simulation tool COMPASS. It aims to estimate various errors on the DM commands at each loop iteration taking into account the AO loop filter. Detailed equations had already been described in Ref. 4. This section summarizes those equations and gives an overview of the error contributors that are estimated. Bold characters will be used to denote vectors.

\subsection{AO loop command error}

Let consider a simple Single Conjugate Adaptive Optics (SCAO) system: a single Wave-Front Sensor (WFS) and a single DM, coupled to a tip-tilt mirror, both conjugated to ground. The residual phase $\Phi \epsilon$ in the scientific camera path of the AO system can be written as:

$$
\Phi_{\epsilon}=\Phi+\Phi_{D M}=\sum_{i}^{N} a_{i} \mathcal{B}_{i}+\Phi_{\perp}+\sum_{i}^{N} v_{i} \mathcal{B}_{i}=\sum_{i}^{N} \epsilon_{i} \mathcal{B}_{i}+\Phi_{\perp}
$$

where:

- $\Phi$ is input turbulent phase

- $\mathcal{B}$ is a basis of the DM space composed by $N$ commanded modes

- $\Phi_{D M}=\sum_{i}^{N} v_{i} \mathcal{B}_{i}$ is the phase produced by the DM shape

- $\sum_{i}^{N} a_{i} \mathcal{B}_{i}=\Phi_{\|}$is the $\Phi$ component that belongs to the DM space

- $\Phi_{\perp}$ is the $\Phi$ component that is orthogonal to the DM space

Usually, AO systems operates in closed loop which means that the WFS measures the residual wave-front. Moreover, in a general case the scientific object cannot be used as a Guide Star (GS) for the WFS because of its low brightness. Then, a bright star is chosen as close as possible of the scientific object to limit the anisoplanatism effect. We note $\hat{\Phi}$ the turbulent phase in the GS direction, i.e. the phase actually seen by the WFS. In the same 
way, we will note with a hat all the variables that refer to the GS direction. Then, considering a linear WFS and a system with a 1-frame delay, its measurements at iteration $k$ can be written as:

$$
\mathbf{M}_{k}=\mathcal{M}\left(\hat{\Phi}_{\|}^{k}\right)+\mathcal{M}\left(\Phi_{D M}^{k-1}\right)+\mathcal{M}\left(\hat{\Phi}_{\perp}^{k}\right)+\mathbf{N}_{k}+\mathbf{S}_{k}
$$

where $\mathcal{M}$ is the linear operator that describes the measurement obtained by the wavefront sensor from an input phase, $\mathbf{N}_{k}$ is the noise on the measurement vector at frame $k$ and $\mathbf{S}_{k}$ is the sensor non-linearity effect, such as undersampling effect or truncation error in a Shack-Hartmann pattern at frame $k$. The term $\mathcal{M}\left(\hat{\Phi}_{\perp}^{k}\right)$ is the aliasing term in the GS direction due to the sampling of the wavefront. We will note this term $\hat{\mathbf{A}}_{k}$. Introducing the interaction matrix $D$ such as $\mathbf{M}=D \mathbf{v}$ that gives the relation between measurement and command $\mathbf{v}$ applied on the DM, Eq. 2 becomes:

$$
\mathbf{M}_{k}=D \hat{\mathbf{a}}_{k}+D \mathbf{v}_{k-1}+\hat{\mathbf{A}}_{k}+\mathbf{N}_{k}+\mathbf{S}_{k}
$$

Considering that the system command law is an integral controller with gain $g$, the command $\mathbf{v}_{k}$ that will be applied to the DM at iteration $k$ is:

$$
\mathbf{v}_{k}=\mathbf{v}_{k-1}-g R \mathbf{M}_{k}
$$

with $R=\left(D^{t} D\right)^{+} D^{t}$ the pseudo-inverse of the interaction matrix. Using Eq. 3 for $\mathbf{M}_{k}$, the command vector $\mathbf{v}_{k}$ to apply at the iteration $k$ can be written as:

$$
\mathbf{v}_{k}=(1-g R D) \mathbf{v}_{k-1}-g R D \hat{\mathbf{a}}_{k}-g R \hat{\mathbf{A}}_{k}-g R \mathbf{N}_{k}-g R \mathbf{S}_{k}
$$

Finally, the AO loop residual error at iteration $k$ is defined by:

$$
\boldsymbol{\epsilon}_{k}=\mathbf{a}_{k}+\mathbf{v}_{k-1}
$$

Using Eq. 5 in this definition, we are able to derive the expression of the AO loop command error:

$$
\boldsymbol{\epsilon}_{k}=(1-g R D) \boldsymbol{\epsilon}_{k-1}+\left(\mathbf{a}_{k}-\mathbf{a}_{k-1}\right)+g R D\left(\mathbf{a}_{k-1}-\hat{\mathbf{a}}_{k-1}\right)-g R \hat{\mathbf{A}}_{k-1}-g R \mathbf{N}_{k-1}-g R \mathbf{S}_{k-1}
$$

We define:

$$
\boldsymbol{\epsilon}_{k}=\mathbf{b}_{k}+\mathbf{t}_{k}+\boldsymbol{\alpha}_{k}+\mathbf{n}_{k}+\mathbf{s}_{k}
$$

Each term of this equation is a contributor to the total error breakdown and can be estimated by ROKET at each iteration of the simulation run. The output of the ROKET simulation run is not only the point-spread function or phase variance, but time-series of all the terms of Eq. 8, namely bandwidth, anisoplanatism, aliasing, noise and non-linearity errors.

\subsection{Error contributors}

In this section we will break the Eq. 7 into 5 equations, each related to the 5 terms cited above. Summing together the equations 9 to 13 brings us back to Eq. 7 .

\subsubsection{Bandwidth error $\mathbf{b}_{k}$}

The second term of the Eq. 7 is a temporal error due to the delay between the time when the command is computed and the time when this command is actually applied on the DM. During the computation, the turbulent phase is evolving whereas the DM shape does not. It can be interpreted as the difference between the actual turbulent phase and the command that it is applied at the same time. Hence, this term is obtained from the same equation as Eq. 6, except that we consider it without any additional error source. We estimate the bandwidth error contribution $\mathbf{b}_{k}$ as:

$$
\mathbf{b}_{k}=(1-g R D) \mathbf{b}_{k-1}+\left(\mathbf{a}_{k}-\mathbf{a}_{k-1}\right)
$$

It should be noticed that this term involves the phase in the direction of the scientific object only. 


\subsubsection{Anisoplanatism error $\mathbf{t}_{k}$}

The third term of the Eq. 7 is an anisoplanatism error due to the wavefront difference between the scientific direction and the analysis direction. This contribution $\mathbf{t}_{k}$ to the error budget is estimated as:

$$
\mathbf{t}_{k}=(1-g R D) \mathbf{t}_{k-1}+g R D\left(\mathbf{a}_{k-1}-\hat{\mathbf{a}}_{k-1}\right)
$$

We notice this anisoplanatism error is estimated in the DM space only, and filtered by the temporal response through the AO loop.

\subsubsection{Noise measurement error $\mathbf{n}_{k}$}

$\mathbf{N}_{k}$ is the noise of the WFS measurements, induced by both read-out and photon noises. The error term $\mathbf{n}_{k}$ due to the impact of the noise on the WFS measurement after loop filtering is estimated as:

$$
\mathbf{n}_{k}=(1-g R D) \mathbf{n}_{k-1}-g R \mathbf{N}_{k-1}
$$

\subsubsection{WFS non-linearity error $\mathrm{s}_{k}$}

$\mathbf{S}_{k}$ describes any non-linear effects of the WFS, such as truncation that could occur if the field of view of subapertures is too small compared to the spot size. This term can also contain undersampling error and leads to an estimation of the error contribution $\mathbf{s}_{k}$ on the error budget:

$$
\mathbf{s}_{k}=(1-g R D) \mathbf{s}_{k-1}-g R \mathbf{S}_{k-1}
$$

\subsubsection{Aliasing error $\alpha_{k}$}

$\hat{\mathbf{A}}_{k}$ is the aliasing term: the high frequencies of turbulent phase are misinterpreted by the WFS and give a nonnull measurement, reconstructed and compensated by the AO system, introducing an aliased phase component. Its impact $\boldsymbol{\alpha}_{k}$ on the residual error is then estimated as:

$$
\boldsymbol{\alpha}_{k}=(1-g R D) \boldsymbol{\alpha}_{k-1}-g R \hat{\mathbf{A}}_{k-1}
$$

Note that this contributor and all the others detailed above are also filtered by the AO loop.

\subsubsection{Fitting and filtered modes error $\Phi_{\perp}$}

Fitting and filtered modes term $\Phi_{\perp}$ remains in the residual phase. The filtered modes error is due to the filtering process used to inverse the interaction matrix. It can be estimated thanks to the right properties of the basis $\mathcal{B}$ that can express controlled modes and filtered modes as orthogonal modes. As the fitting is orthogonal to the commanded DM space, it cannot be expressed as an AO loop error term. In order to estimate impact on Strehl ratio, we compute at each iteration the phase orthogonal to the DM space and, then, derive its mean spatial variance.

\section{CORRELATIONS ANALYSIS}

This section aims to study correlations that could potentially exist between some of the error contributors in an ELT simulation case. Those correlations are easily derivable from the ROKET outputs. 


\subsection{Definition}

As ROKET outputs are temporal buffers of each error contributors computed at each iteration, the computation of the covariance matrix of each error term is straightforward. Let $\mathbf{x}$ and $\mathbf{y}$ be two of the temporal buffers computed by ROKET, representing two terms of the error contributors. The covariance matrix $C_{x y}$ between $\mathrm{x}$ and $\mathbf{y}$ is:

$$
C_{x y}=\left\langle\mathbf{x} \cdot \mathbf{y}^{t}\right\rangle
$$

Moreover, if those buffers are expressed in a orthonormal modal basis that span the full DM space, the global covariance $\sigma_{x y}^{2}$ between the two contributors is the sum of the diagonal elements of the covariance matrix:

$$
\sigma_{x y}^{2}=\sum_{k=1}^{N} C_{x y}(k, k)
$$

Finally, we can define the correlation coefficient $r_{x y}$ between those two contributors as:

$$
r_{x y}=\frac{\sigma_{x y}^{2}}{\sqrt{\sigma_{x x}^{2} \sigma_{y y}^{2}}}
$$

\subsection{Simulation case}

\begin{tabular}{|c|c|c|c|}
\hline \multicolumn{2}{|c|}{ Telescope parameters } & \multicolumn{2}{|l|}{ Target parameters } \\
\hline Diameter & $39 \mathrm{~m}$ & Wavelength $\lambda_{t}$ & $1.65 \mu \mathrm{m}$ \\
\hline \multicolumn{2}{|c|}{ Atmospheric parameters } & \multicolumn{2}{|l|}{$\begin{array}{l}\text { WFS parameters } \\
\end{array}$} \\
\hline$r_{0} @ 0.5 \mu m$ & $0.16 \mathrm{~m}$ & Number of sub-apertures & $78 \times 78$ \\
\hline Number of layers & 1 & Wavelength $\lambda_{w f s}$ & $0.5 \mu m$ \\
\hline Layer altitude & $2000 \mathrm{~m}$ & Number of pixels per sub-aperture & 6 \\
\hline Windspeed & $10 \mathrm{~m} \mathrm{~s}^{-1}$ & Pixel angular size & $0.5 \%$ \\
\hline \multirow[t]{3}{*}{ Wind direction } & $45^{\circ}$ & Photons per sub-aperture per frame & 76000 \\
\hline & & Guide star coordinates in FoV & $(5 ", 0 ")$ \\
\hline & & Readout noise & $3 e^{-}$ \\
\hline \multicolumn{2}{|c|}{ AO parameters } & \multicolumn{2}{|l|}{ DM parameters } \\
\hline Loop frequency & $500 \mathrm{~Hz}$ & Number of actuators & $79 \times 79$ \\
\hline Command law & Integrator & Conjugate altitude & $0 \mathrm{~m}$ \\
\hline Loop gain & 0.3 & 1 tip-tilt DM & \\
\hline Delay & 1 frame & & \\
\hline Frames & 40000 & & \\
\hline
\end{tabular}

We consider a $39 \mathrm{~m}$ diameter telescope without central obstruction. The parameters used are listed in the Table 1. This case was simulated over 40,000 iterations, equivalent to 80 seconds of observation. The coordinates and

directions are defined in a reference frame where the centre is the science target and the X-axis is oriented towards the WFS guide star. Note that even if the following results are given for a single turbulent layer simulation, they remain valid for a complex profile as the turbulence is composed of individual statistically independent layers. Then, the total error breakdown for the turbulent contributors is linear and can be summed through the turbulent layers. The noise term is finally added after this sum.

\subsection{Correlations}

Using Eqs. 14, 15, 16, we can compute the covariances and correlation coefficients between all the error breakdown contributors estimated by ROKET for the simulation case described above. Figure 1 represents the results as a matrix, the cell intersecting each row $x$ and column $y$ corresponding to the error contributor $\sigma_{x y}^{2}$. Note that the 


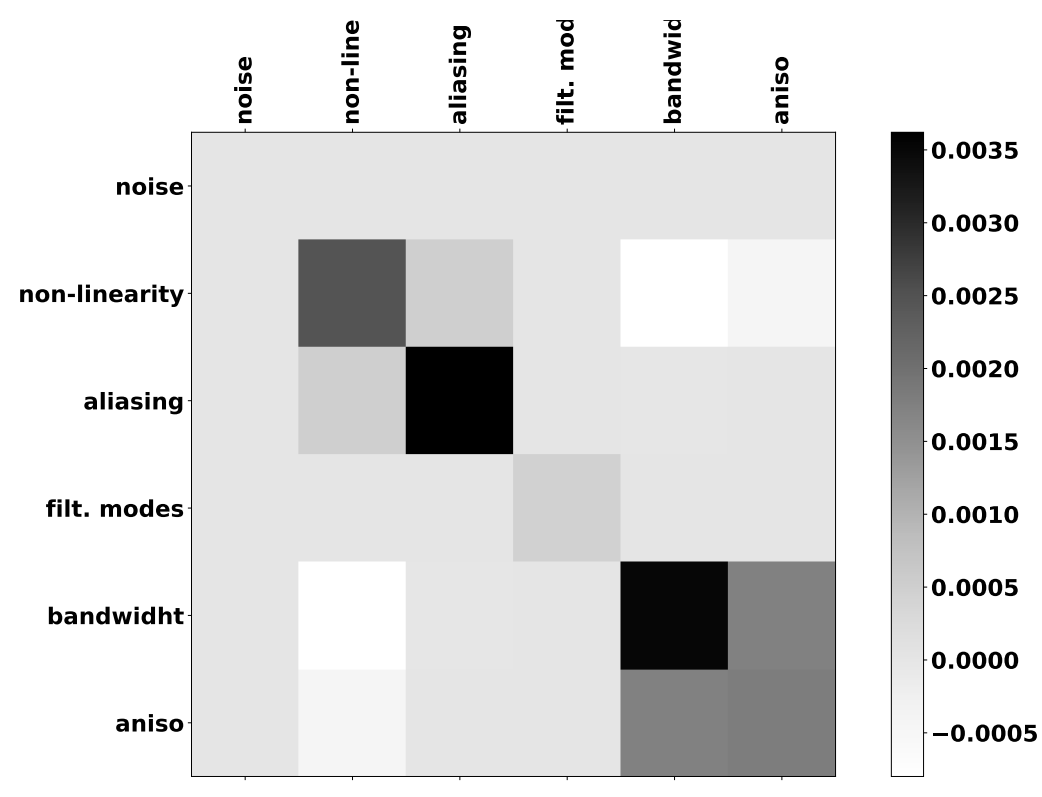

(a)

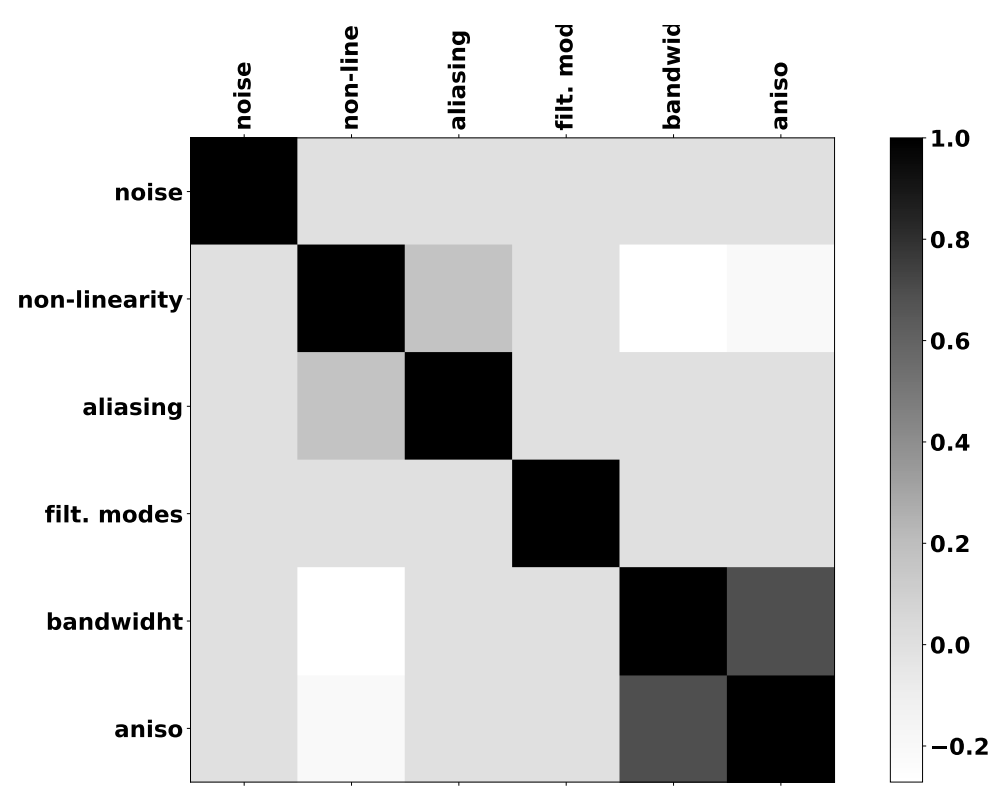

(b)

Figure 1. (a) Covariance between the error contributors expressed in $\mu m^{2}$, (b) Correlation coefficients between the error contributors

total error wavefront variance is equal to the sum of all the elements of this matrix. The fitting error is obviously missing in those matrices as it cannot be expressed in the DM space. So, it is supposed to be orthogonal to the other error contributors. However, ROKET can estimate the fitting error from the residual phase error. Then, ROKET can provide the full error breakdown as shown in Table 2 and that corresponds to the diagonal of the matrix shown in Figure 1(a). 


\begin{tabular}{cc}
\hline \hline Contributors & $\sigma$ [nm rms] \\
\hline Fitting & 114 \\
Anisoplanatism & 42 \\
Bandwidth & 60 \\
Aliasing & 60 \\
Noise & 1 \\
Non linearity & 50 \\
Filtered modes & 21
\end{tabular}

Table 2. Error breakdown returned by ROKET for the test case

From Figure 1(b), we notice that a strong correlation of about 0.70 exists between anisoplanatism and bandwidth error. Significant correlations also exist between aliasing and WFS non linearity errors, and between WFS non linearity, bandwidth and anisoplanatism. In this paper, we will focus only on the largest correlation observed, i.e. between anisoplanatism and bandwidth errors. A more detailed analysis of all others correlations will be discussed in Ref. 6.

The correlation between anisoplanatism and bandwidth is due to the motion of the turbulent layer and the loop delay. It is positive correlation if the wind direction is along the GS direction, it is an anti-correlation in the other case. It could be important to take this effect into account for the PSF reconstruction, as shown in Ref. 4. The effect on the PSF estimation is obviously weighted by the importance of those contributors in the overall error breakdown, but also by the turbulent profile. As anisoplanatism is null on the ground layer which is usually the strongest one, the correlation observed in a more realistic simulation with a multi-layer profile will be lower. Random directions of wind through all the layers tend also to decrease this correlation factor.

Nevertheless, we want to propose a way to model analytically the possible correlation between anisoplanatism and bandwidth errors. As those errors term often influence a lot the error breakdown of an AO system and so its PSF, such a model allows to estimate an accurate PSF of the system.

\section{BANDWIDTH \& ANISOPLANATISM ERROR MODEL}

In this section, we expose our model, called GROOT (Gpu-based Residual errOr cOvariance maTrix), that we have developed to handle bandwidth, anisoplanatism errors and their correlation. More details on this model and its implementation will be available in Ref. 6 . After the description, another section will present some results obtained with GROOT on the simulation case of Table 1.

\subsection{GROOT}

GROOT is a pseudo analytical model that aims to produce the covariance matrix of the AO system residual error. This matrix is expressed in the DM command space and can be then used for PSF estimation. ${ }^{1,7}$ Thanks to this model coupled with a PSF reconstruction algorithm, we are able to produce accurate synthetic PSF from the knowledge of the system and the turbulent profile.

The principle of the model is to express the bandwidth and anisoplanatism errors both as spatial errors and the covariance matrix of those errors as a sum of structure function. This structure function used by in the model is filtered from spatial frequencies higher than maximum frequency that the DM can reach. Hence, GROOT computes the covariance matrix of the anisoplanatism error $C_{a a}$, the one of the bandwidth error $C_{b b}$ and the covariance matrix of bandwidth with anisoplanatism error $C_{b a}$ to take into account eventual correlation. Those matrices are computed as:

$$
\begin{aligned}
C_{a a}(i, j) & =\frac{1}{2}\left(D_{\phi}^{\text {low }}\left(\overrightarrow{x_{i j}}+h \vec{\theta}\right)+D_{\phi}^{\text {low }}\left(\overrightarrow{x_{i j}}-h \vec{\theta}\right)-2 D_{\phi}^{\text {low }}\left(\overrightarrow{x_{i j}}\right)\right) \\
C_{b b}(i, j) & =\frac{1}{2}\left(D_{\phi}^{l o w}\left(\overrightarrow{x_{i j}}+\vec{v} \tau\right)+D_{\phi}^{l o w}\left(\overrightarrow{x_{i j}}-\vec{v} \tau\right)-2 D_{\phi}^{l o w}\left(\overrightarrow{x_{i j}}\right)\right) \\
C_{b a}(i, j) & =\frac{1}{2}\left(-D_{\phi}^{l o w}\left(\overrightarrow{x_{i j}}-h \vec{\theta}-\vec{v} \tau\right)+D_{\phi}^{l o w}\left(\overrightarrow{x_{i j}}-h \vec{\theta}\right)+D_{\phi}^{l o w}\left(\overrightarrow{x_{i j}}-\vec{v} \tau\right)-D_{\phi}^{l o w}\left(\overrightarrow{x_{i j}}\right)\right)
\end{aligned}
$$

where: 
- $D_{\phi}^{\text {low }}$ is the modified structure function

- $\vec{x}_{i j}$ is the separation vector between actuators $i$ and $j$ of the DM

- $h$ is the layer altitude

- $\vec{\theta}$ is the angular separation vector between the GS and the scientific direction

- $\vec{v}$ is the wind velocity vector

- $\tau$ is the sampling period divided by the loop gain

Finally, the total covariance matrix $C_{e e}$ due to anisoplanatism and bandwidth errors is obtained as:

$$
C_{e e}=C_{b b}+C_{a a}+C_{b a}+C_{b a}^{t}
$$

\subsection{Results}

As mentioned above, the error covariance matrix can be used to reconstruct a PSF. To verify the accuracy of the GROOT model, we can reconstruct a PSF from the synthetic matrix $C_{e e}$ and compare it to a PSF obtained from the same matrix directly computed from the ROKET outputs. Figure 2 shows those PSFs and their difference with logarithmic scale. The PSF obtained from the GROOT model reaches a Strehl ratio of $87.6 \%$ where the one directly obtained from the ROKET outputs is $88.0 \%$. And the ensquared energy at $5 \lambda / \mathrm{D}$ is equal to 88.2 $\%$ with the model compared to $88.3 \%$. Indeed, the PSF obtained with the GROOT model (Fig. 2 (b)) well reproduces the structure of the PSF obtained from ROKET (Fig. 2 (a)). This is confirmed by the Fig. 2(c) where we can observe that the residual difference is mainly due to the convergence of simulation that gives us the PSF in Fig. 2 (a) whereas the GROOT model is based on a mathematical expectation of the error covariance.

\subsection{Computation performance}

A great advantage of this model is that it is highly parallelizable because Eqs. 17 to 19 allow us to compute each matrix coefficient in an independent way from the others ; they could all be computed in parallel. Then, using GPU acceleration, the GROOT model can compute the covariance matrix very efficiently. The CUDA implementation used takes advantage of GPU optimized library such CuBLAS or CuFFT. For this ELT case, we have ran it on a Nvidia DGX-1 server with a dual 20-core Intel Xeon E5-2698 v4 @ 2.2GHz and 8 Tesla GP100. This environment allowed us to compute the 4900 by $4900 C_{e e}$ matrix in less than a second.

Moreover, we have also implemented a GPU version of the PSF reconstruction algorithm described in Ref. 7. This GPU module, called GAMORA (Gpu Accelerated Module fOr psf Reconstruction Algorithms), is able to reconstruct a 4096 by 4096 pixels PSF over 4900 modes in 33 seconds using 4 GPUs. Hence, by coupling GROOT and GAMORA, we can estimate a PSF at ELT scale in about 34 seconds.

\section{CONCLUSION \& PERSPECTIVES}

We have used the error breakdown estimation provided by ROKET (erROr breaKdown Estimation Tool) to derive and validate a pseudo analytical model of the anisoplanatism and bandwidth errors, including the possible correlation that could exist between them. The model only requires a few parameters from the AO system geometry and from the turbulent profile. It reaches excellent performance in terms of accuracy for PSF reconstruction, and also in terms of computing performance even at an ELT scale.

Future developments will study ways to model the other contributors (aliasing, noise, non-linearities) of the error breakdown in order to include those errors in the GROOT (Gpu-based Residual errOr cOvariance maTrix) model. The goal of these studies is twofold. First it will allow us fast and accurate PSF estimation of the performance of an ELT AO system. Second, they will form a starting basis for exploring ways to retrieve the parameters needed to model the PSF from on-sky WFS data. 


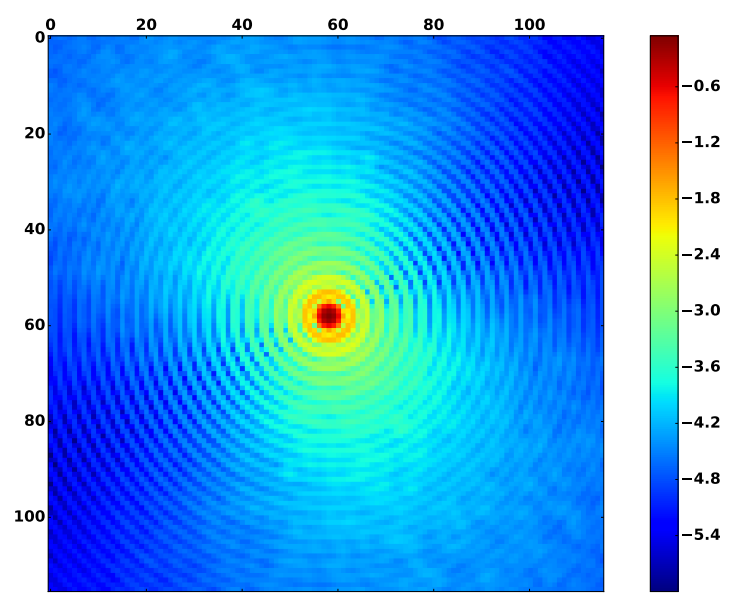

(a)

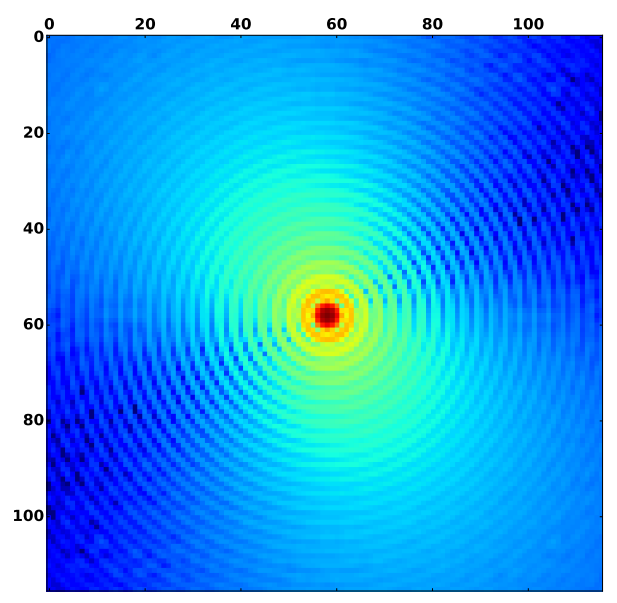

(b)

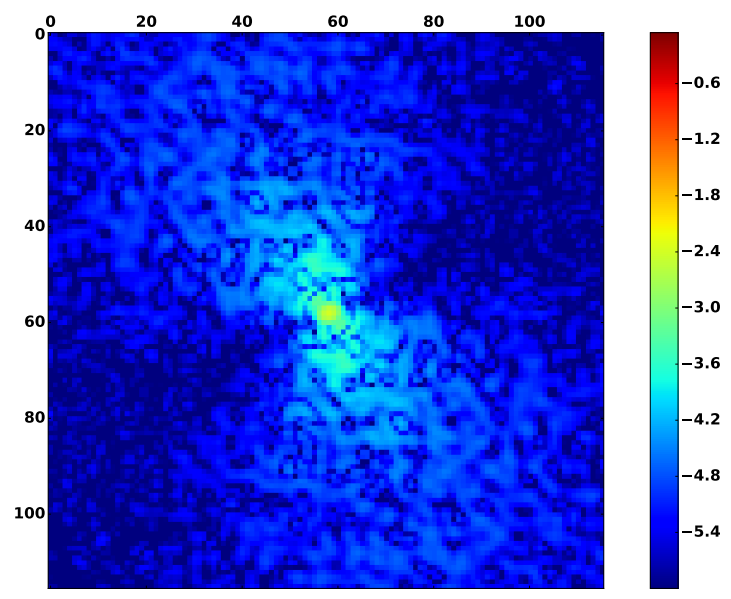

(c)

Figure 2. (a) PSF due to anisoplanatism and bandwidth errors obtained from ROKET outputs, (b) PSF due to anisoplanatism and bandwidth errors obtained from GROOT model, (c) Difference between the two PSFs. In all images, width is $40 \lambda / \mathrm{D}$ 


\section{ACKNOWLEDGMENTS}

This work is sponsored through a grant from project \#671662, a.k.a. Green Flash, funded by European Commission under program H2020-EU.1.2.2 coordinated in H2020-FETHPC-2014

\section{REFERENCES}

[1] Veran, J.-P., Rigaut, F., Maitre, H., and Rouan, D., "Estimation of the adaptive optics long-exposure pointspread function using control loop data.," J. Opt. Soc. Am. A, Vol. 14, No. 11, p. 3057 - 3069 14, 3057-3069 (Nov. 1997).

[2] Correia, C., Véran, J.-P., Ellerbroek, B., Gilles, L., and Wang, L., "Laser-Guide Star Point-Spread Function Reconstruction for ELTs," in [Second International Conference on Adaptive Optics for Extremely Large Telescopes], 70 (Sept. 2011).

[3] Gendron, É., Charara, A., Abdelfattah, A., Gratadour, D., Keyes, D., Ltaief, H., Morel, C., Vidal, F., Sevin, A., and Rousset, G., "A novel fast and accurate pseudo-analytical simulation approach for MOAO," in [Adaptive Optics Systems IV], Proc. SPIE 9148, 91486L (Aug. 2014).

[4] Ferreira, F., Gendron, E., Rousset, G., and Gratadour, D., "Deriving comprehensive error breakdown for wide field adaptive optics systems using end-to-end simulations," in [Adaptive Optics Systems V], Proc. SPIE 9909, 990979 (July 2016).

[5] Gratadour, D., Puech, M., Vérinaud, C., Kestener, P., Gray, M., Petit, C., Brulé, J., Clénet, Y., Ferreira, F., Gendron, E., Lainé, M., Sevin, A., Rousset, G., Hammer, F., Jégouzo, I., Paillous, M., Taburet, S., Yang, Y., Beuzit, J.-L., Carlotti, A., Westphal, M., Epinat, B., Ferrari, M., Gautrais, T., Lambert, J. C., Neichel, B., and Rodionov, S., "COMPASS: an efficient, scalable and versatile numerical platform for the development of ELT AO systems," in [Adaptive Optics Systems IV], Proc. SPIE 9148, 91486 O (Aug. 2014).

[6] Ferreira, F., Gendron, E., Rousset, G., and Gratadour, D., "To be published," $A \mathscr{E} A$ A (2018).

[7] Gendron, E., Clénet, Y., Fusco, T., and Rousset, G., "New algorithms for adaptive optics point-spread function reconstruction," $A \& A 457,359-363$ (Oct. 2006). 